Research Article

\title{
Diversity of Mycobacterium tuberculosis Complex Lineages Associated with Pulmonary Tuberculosis in Southwestern, Uganda
}

\author{
Lisa Nkatha Micheni, ${ }^{1,2}$ Kennedy Kassaza, ${ }^{1}$ Hellen Kinyi, ${ }^{3}$ Ibrahim Ntulume, ${ }^{2}$ \\ and Joel Bazira $\mathbb{1 D}^{1}$ \\ ${ }^{1}$ Department of Microbiology, Mbarara University of Science and Technology, P.O. Box 1410 Mbarara, Uganda \\ ${ }^{2}$ Department of Microbiology and Immunology, Kampala International University Western Campus, P.O. Box 71, Bushenyi, Uganda \\ ${ }^{3}$ Department of Biochemistry, School of Medicine, Kabale University, P.O. Box 317, Kabale, Uganda \\ Correspondence should be addressed to Joel Bazira; jbazira@gmail.com
}

Received 1 March 2021; Accepted 5 June 2021; Published 28 June 2021

Academic Editor: Adwoa Asante-Poku

Copyright ( 2021 Lisa Nkatha Micheni et al. This is an open access article distributed under the Creative Commons Attribution License, which permits unrestricted use, distribution, and reproduction in any medium, provided the original work is properly cited.

\begin{abstract}
Uganda is among the 22 countries in the world with a high burden of tuberculosis. The southwestern region of the country has consistently registered a high TB/HIV incidence rate. This study is aimed at characterizing the Mycobacterium tuberculosis complex (MTBC) genotypic diversity in southwestern Uganda. A total of 283 sputum samples from patients with pulmonary tuberculosis were genotyped using specific single nucleotide polymorphism markers for lineages 3 and 4 . Most of the patients were males with a mean age of 34 . The lineage 4 Ugandan family was found to be the most dominant strains accounting for $59.7 \%$ of all cases followed by lineage 3 at $15.2 \%$. The lineage 4 non-Ugandan family accounted for $14.5 \%$ of all cases while $4.2 \%$ showed amplification for both lineage 4 and lineage 3. Eighteen samples (6.4\%) of the strains remained unclassified since they could not be matched to any lineage based on the genotyping technique used. This study demonstrates that a wide diversity of strains is causing pulmonary tuberculosis in this region with those belonging to the lineage 4 Ugandan family being more predominant. However, to confirm this, further studies using more discriminative genotyping methods are necessary.
\end{abstract}

\section{Introduction}

Tuberculosis (TB) is an ancient communicable disease that has been in existence over the past millennia and up to date remains a major global public health problem. It is caused by closely related acid-fast bacteria known as Mycobacterium tuberculosis complex (MTBC) with seven human-adopted MTBC lineages (designated lineage 1 through lineage 7 ) and two lineages adapted to various wild and domestic animal species but capable of causing human infection being implicated [1-4]. Epidemiological studies reveal that there is a strong phylogeographical structuring of this organism [5-7] and well adapted to sympatric human populations [8, 9]. This makes these lineages more predominant in specific human populations of certain geographical locations $[1,7$, 10]. For instance, lineage 2 (L2) is most predominant in East
Asia and is more associated with virulence and drug-resistant than other lineages $[11,12]$. L1 (moderately virulent) and L3 mainly occur in areas around the Indian Ocean whereas L5 and L6 are highly restricted to West Africa [13, 14]. L4 is commonly found in Africa, Central America, Europe, and South America [15], while L7 is highly and exclusively restricted in Ethiopia [16]. This adoption could be a result of coevolution $[17,18]$. Studies have also shown that human migration greatly contributes to the spread of $\mathrm{TB}$ and causes an increase in the genetic diversity of MTBC $[9,19-23]$.

Uganda is listed among the world's 22 countries with the highest TB burden countries [24] and the third-largest refugee host country in the world after Turkey and Pakistan with over 1.36 million refugees [25-27]. The refugees come from countries such as the Democratic Republic of the Congo (DRC), Burundi, Ethiopia, Eritrea, Rwanda, Somalia, and 
South Sudan. Over the past five years, there has been a mass refugee influx from DRC and South Sudan into the country with the southwestern region of the country accommodating most of these refugees. Ninety-two percent (92\%) of these refugees live alongside the local communities where accommodation and farming land are given to them [27]. Earlier studies conducted in central Uganda indicate that majority of the TB cases are due to MTBC lineage 4 sublineage Ugandan family (L4-U) [28-30], Euro-American lineage. This sublineage is defined by a deletion in 724 region of difference (RD) and (SNP) typing 33-36, 40 and 43 spoligotype fingerprint spacers missing, and several single nucleotide polymorphisms (SNPs). Similarly, a 2010 study in southwestern Uganda done by Bazira and colleagues using spoligotyping indicated that the majority of TB strains in the region belong to the Uganda genotype [31]. However, the fact that there has been an influx of immigrants in a region that consistently registers high TB incidence rates calls for a greater understanding of the molecular epidemiology and genetic variability of MTBC in the region to enable better control of this causative agent of TB.

\section{Methods}

2.1. Study Setting. This study was conducted between May 2018 and April 2019 in the southwestern region of Uganda, a region with fifteen administrative districts and borders Tanzania, Rwanda, and the Democratic Republic of Congo (Supplementary File 1: Figure 1). The region is heavily affected by the TB/HIV epidemic and constantly registers high TB incidence rates [32-34]. There were four patient recruitment centers which included two regional referral hospitals (Kabale and Mbarara regional referral hospitals) and two health centers within the refugee camps in the region (Oruchinga and Nakivale health center IV).

2.2. Patient Recruitment and Sample Collection. The patient's clinical information including age, sex, HIV status, previous history of TB, and economic status was recorded. Sputum samples were collected from patients aged $\geq 18$ years. Cases of pulmonary tuberculosis (PTB) were diagnosed at the sample collection centers by either GeneXpert Cepheid test for samples analyzed at the regional referral hospitals or sputum smear microscopy for samples analyzed in the health centers. Those samples diagnosed positive for PTB were then transported (not more than 72 hours after collection time) in a cold box to Mbarara University of Science and Technology Genomics and Translational Laboratory for processing and molecular analysis.

2.3. DNA Extraction and Confirmation of MTB in Sputum Samples. The genomic DNA from each patient sample was processed by standardized protocols $[35,36]$. All the samples were then screened and confirmed as MTB by detection of a $123 \mathrm{bp}$ fragment of the IS6110 gene which is common among the members of the MTBC.

2.4. Single Nucleotide Polymorphism (SNP) Typing. SNP typing to determine the MTB lineages was performed by RT-PCR (Bio-Rad CFX96 Touch ${ }^{\mathrm{TM}}$ ). Lineage-specific primers were as follows: Rv004C for MTB L4-U, Rv2962C for MTB L4-NU, and Rv0129C for MTB L3 based on a previous study of Wampande et al. [37] and their accompanying hybridization probes (Supplementary File 2: Table 1). The MTBC lineages were identified based on differences in melting temperature $\left(T_{\mathrm{m}}\right)$. Briefly, the assays were performed in 20 $\mu \mathrm{l}$ reaction mixture containing $3.75 \mu \mathrm{l}$ of PCR water, $1.25 \mu \mathrm{l}$ $(0.5 \mu \mathrm{M}$ final concentration) of each primer, $0.625 \mu \mathrm{l}(0.25$ $\mu \mathrm{M}$ final concentration) of each probe, $9.5 \mu \mathrm{l}$ of $2 \mathrm{X}$ Lunar $^{\circledR}$ Universal genotyping master mix, and $3 \mu \mathrm{l}$ (5-50 ng) of extracted genomic DNA. The Bio-Rad CFX96 Touch $^{\mathrm{TM}}$ Real-Time PCR Detection System was programmed for PCR amplification and a melting curve stage. For each of the three uniplex assays, the amplification stage consisted of a pre-PCR stage performed at $95^{\circ} \mathrm{C}$ for $10 \mathrm{~min}$, an amplification stage with denaturation at $95^{\circ} \mathrm{C}$ for $30 \mathrm{~s}$, primer annealing $\left(50^{\circ} \mathrm{C}\right.$ for Rv004C or $52^{\circ} \mathrm{C}$ for Rv0129C or $51^{\circ} \mathrm{C}$ for Rv2962C) for $30 \mathrm{~s}$, and extension at $60^{\circ} \mathrm{C}$ for $30 \mathrm{~s}$ for 45 cycles. The melting curve analysis consisted of denaturation of the amplicons at $95^{\circ} \mathrm{C}$ for $10 \mathrm{~s}$ to produce single-stranded DNA, probe annealing temperature at $65^{\circ} \mathrm{C}$ for $05 \mathrm{~s}$ with a continuous acquisition mode to allow capture of the fluorescence, and probe melting temperature ranging from 40 to $80^{\circ} \mathrm{C}$. In all the assays, kc32969 (L4-U), H37Rv (L4-NU), and delicus (L3) genomic DNA (courtesy of Makerere Molecular Labs) were used as positive control while nontemplate mix as a negative control. The Bio-Rad CFX96 Touch $^{\text {TM }}$ software was used to determine the MTB lineages through the analysis of amplicon melting temperature $\left(T_{\mathrm{m}}\right)$ (Supplementary File 3: Figure 2).

2.5. Statistical Analysis. Patient demographic data were converted into Excel tables and then exported to SPSS version 25 (IBM, Chicago, USA) for analysis. Bivariate analysis was performed using the chi-square test to determine the relationship between categorical variables (independent variables) and dependent variables. Multinomial logistic regression models were fitted to evaluate the relationship between MTB lineage (dependent variable) and patients' country of origin/ethnicity (primary independent variable). Patients' characteristics of age, gender, and economic status were treated as covariates when fitting the final model. Statistical significance was considered at $p \leq 0.05$. Chi-square and Fisher's exact tests were computed, and a $p$ value of $\leq 0.05$ was considered evidence of a significant difference.

\section{Results}

A total of 283 samples were genotyped. Majority of the samples $(246,86.9 \%)$ were obtained from Ugandan patients. The mean age of the patients was 34 years, and most $(73.1 \%)$ were males. One hundred and twenty-nine (45.6\%) of the patients had unknown serostatus at the time of the study and did not consent to test while $26.9 \%$ were HIV seropositive. Among the 283 samples analyzed, $59.7 \%$ (169) belonged to lineage 4 Ugandan family (L4-U), 14.5\% (41) were other lineage 4 non-Ugandan (L4-NU), and $15.2 \%$ (43) lineage 3 (L3) while $4.2 \%$ (12) had amplification for both lineage 4 and lineage 3. The remaining $18(6.4 \%)$ strains could not be matched to any 


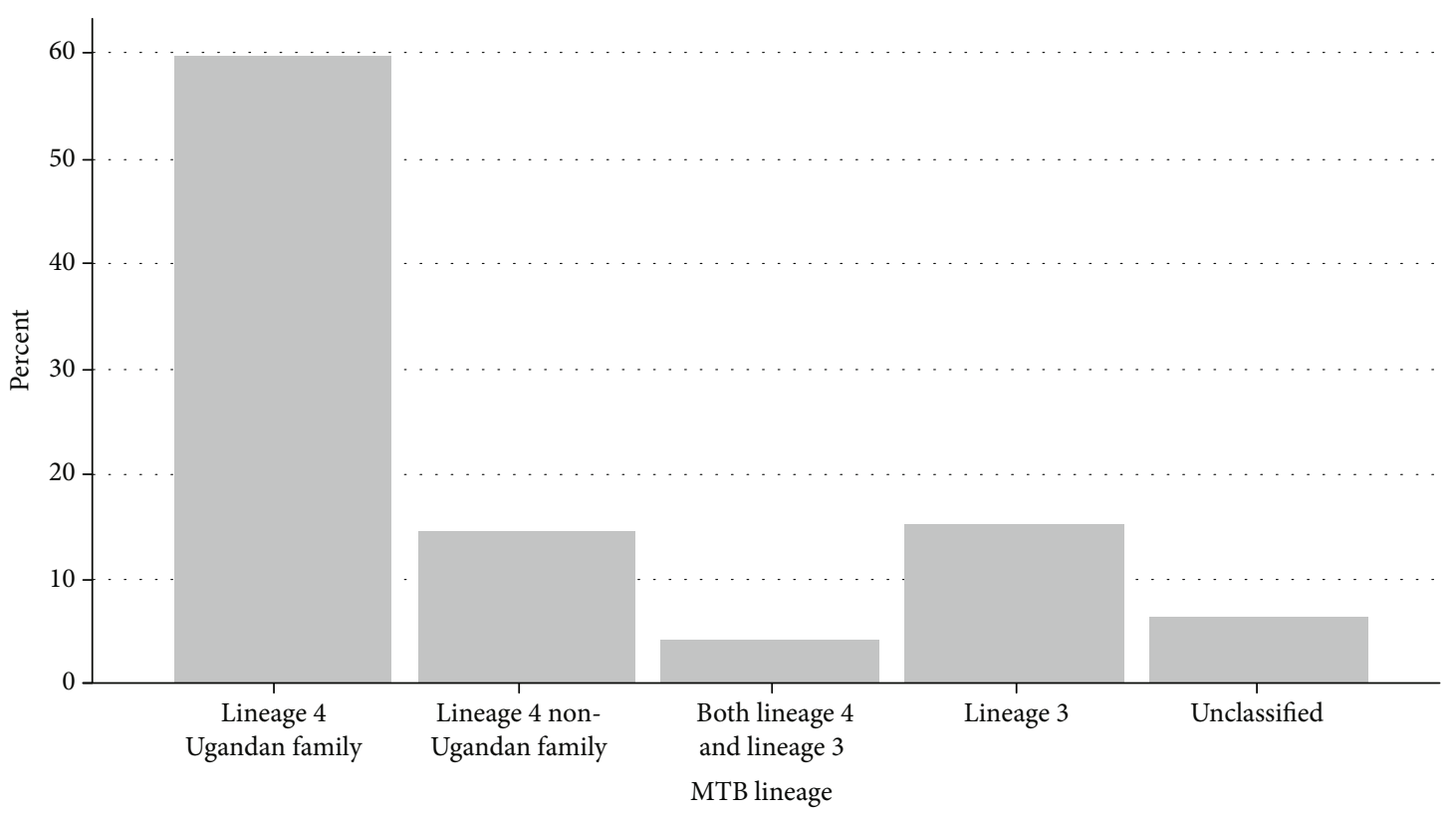

FIGURE 1: Overall prevalence of MTBC lineages causing PTB in southwestern Uganda.

lineage based on the genotyping techniques used (Figure 1). Of the 37 samples from the refugees, $8.1 \%$ belonged to L4U family while $1.1 \%, 3.2 \%$, and $0.4 \%$ were L4-NU, L3, and unclassified strains, respectively. In those samples from the Ugandan patients, $51.6 \%$ belonged to L4-U family while $13.4 \%, 12 \%$, and $6 \%$ were L4-NU, L3, and unclassified strains, respectively (Table 1 ).

Bivariate analysis taking lineage as the outcome showed that the proportions of patients infected with each MTBC lineage did not differ according to age, sex, HIV status, level of income, or history of TB in the past. Despite the fact that the Ugandan genotype was found in a higher proportion of those confirmed by GeneXpert or microscopy, the proportion was not statistically significant $(p=0.074)$ (Table 1$)$. A multinomial logistic regression analysis was further performed to determine the relationship between the independent variable "patients' nationality" and the MTB lineages circulating in southwestern Uganda (Table 2). It was revealed that the Ugandan patients were significantly likely to have L4-U strains (OR: 0.501; 95\% CI: 0.143-1.758; $p$ value: 0.281 ) than the refugee patients when other factors were held constant. Furthermore, the model projected those Ugandan patients were less likely to have strains of lineage 3 $(\mathrm{OR}=0.298)$, both lineages 3 and $4(\mathrm{OR}=0.868)$ than the non-Ugandan patients compared to having lineage 4 nonUgandan family (reference category); however, this was not statistically significant $(p>0.05)$. Conversely, the study showed that Ugandan patients were 1.342 times more likely to have the unclassified strains (95\% CI: $0.130-13.54)$ than the refugee patients when other factors were held constant, but this was not statistically significant $(p>0.05)$.

\section{Discussion}

To gain insight into the MTBC population structure causing PTB in southwestern Uganda, a region where TB infection is widespread, we utilized SNP typing to analyze 283 sputum samples from Ugandan and non-Ugandan patients diagnosed with PTB. SNP typing was chosen since it was optimized and found to be discriminative by Wampande and colleagues [37]. Our findings revealed heterogeneity of MTBC causing PTB in this area, with L4 being the most predominant lineage, with L4-U family and L4-NU accounting for $59.7 \%$ and $14.5 \%$ of all cases, respectively. The L4-U family was represented by $51.6 \%$ of Ugandan and $8.1 \%$ of nonUgandan patients while L4-NU was represented by $13.4 \%$ and $1.1 \%$, respectively. Our results of L4 being the most prevalent in this study are in keeping with reports that indicate TB epidemic in Africa is primarily caused by L4 strains [15]. L4 is phylogenetically complex, with at least ten distinct sublineages that differ in geographical distribution, with local genotypes accounting for a larger proportion of circulating strains in certain regions [15]. For instance, the L4Cameroon family is almost exclusively found in West Africa [14] while the Latin American-Mediterranean (LAM) family is most predominant in Zambia $[38,39]$. Similarly, the L4-U family is more predominant in Uganda as opposed to anywhere else $[4,28,31,40]$. This is supported by our findings which show that L4-U is most common among the Ugandan patients, as opposed to the refugees and comparable with the $63 \%$ recorded in central Uganda [28] and 59.2\% reported in an earlier study conducted in Mbarara, southwestern Uganda [31]. Studies indicate that in neighboring countries, the L4-U family is less common. Tanzania, for example, reported $21.8 \%$ [40] of L4-U, while Kenya reported $11 \%$ [41]. It is thus tempting to speculate that local strains are more likely to transmit in a given local setting compared to others.

Another lineage observed in this study at a relatively high proportion was L3, with a prevalence of $15.2 \%$. The Ugandan patients accounted for $12 \%$ of this percentage while nonUgandans accounted for $3.2 \%$. This finding is comparable to the results of a study conducted in central Uganda, which 
TABLE 1: Distribution of patient variables across the MTB lineages in southwestern Uganda.

\begin{tabular}{|c|c|c|c|c|c|c|c|c|}
\hline Variable & Category & $\begin{array}{c}\text { Patient's } \\
\text { characteristics } n(\%)\end{array}$ & $\begin{array}{c}\mathrm{L} 4-\mathrm{U} \\
(n=169)\end{array}$ & $\begin{array}{l}\mathrm{L} 4-\mathrm{NU} \\
(n=41)\end{array}$ & $\begin{array}{l}\text { MTB lineage } \\
\text { Both L4 and L3 } \\
\quad(n=12)\end{array}$ & $\begin{array}{c}\mathrm{L} 3 \\
(n=43)\end{array}$ & $\begin{array}{l}\text { Unclassified } \\
\qquad(n=18)\end{array}$ & $p$ value* \\
\hline \multirow{2}{*}{ Gender } & Male & $207(73.1)$ & $120(42.4)$ & $34(12)$ & $9(3.2)$ & $32(11.3)$ & $12(4.2)$ & \multirow{2}{*}{0.586} \\
\hline & Female & $76(26.9)$ & $49(17.3)$ & $7(2.5)$ & $3(1.1)$ & $11(3.9)$ & $6(2.1)$ & \\
\hline \multirow{3}{*}{ Age } & $18-45$ years & 209 (73.9) & $125(44.2)$ & $29(10.2)$ & $9(3.2)$ & $33(11.7)$ & $13(4.6)$ & \multirow{3}{*}{0.944} \\
\hline & $46-65$ years & $53(18.7)$ & $29(10.2)$ & $9(3.2)$ & $3(1.1)$ & $8(2.8)$ & $4(1.4)$ & \\
\hline & $\geq 66$ years & $21(7.4)$ & $15(5.3)$ & $3(1.1)$ & $0(0)$ & $2(0.8)$ & $1(0.4)$ & \\
\hline \multirow{3}{*}{ HIV status } & Positive & $76(26.9)$ & $42(14.8)$ & $12(4.2)$ & $4(1.4)$ & $14(4.9)$ & $4(1.4)$ & \multirow{3}{*}{0.813} \\
\hline & Negative & $78(27.6)$ & $51(18.0)$ & $9(3.2)$ & $2(0.7)$ & $9(3.2)$ & $7(2.5)$ & \\
\hline & Unknown & $129(45.6)$ & $76(26.9)$ & $20(7.1)$ & $6(2.1)$ & $20(7.1)$ & $7(2.5)$ & \\
\hline \multirow{2}{*}{ Nationality } & Ugandan & $246(86.9)$ & $146(51.5)$ & $38(13.4)$ & $11(3.9)$ & $34(12.0)$ & $17(6.0)$ & \multirow{2}{*}{0.319} \\
\hline & Non-Ugandan & $37(13.1)$ & $23(8.1)$ & $3(1.1)$ & $1(0.4)$ & $9(3.2)$ & $1(0.4)$ & \\
\hline \multirow{2}{*}{ Income status } & High & $23(8.1)$ & $13(4.6)$ & $4(1.4)$ & $1(0.4)$ & $4(1.4)$ & $1(0.4)$ & \multirow{2}{*}{0.980} \\
\hline & Low & $260(91.9)$ & $156(55.1)$ & $37(13.1)$ & $11(3.9)$ & $39(13.8)$ & $17(6.0)$ & \\
\hline \multirow{2}{*}{$\mathrm{TB}$ in the past } & No & $266(94)$ & $156(55.1)$ & $39(13.8)$ & $12(4.2)$ & $41(14.5)$ & $18(6.4)$ & \multirow{2}{*}{0.503} \\
\hline & Yes & $17(6)$ & $13(4.6)$ & $2(0.7)$ & $0(0)$ & $2(0.7)$ & $0(0)$ & \\
\hline \multirow{2}{*}{ PTB diagnostic results } & High/>5AFB/field & $148(52.3)$ & $82(29)$ & $19(6.7)$ & $7(2.5)$ & $31(11)$ & $9(3.2)$ & \multirow{2}{*}{0.074} \\
\hline & Low/ $\leq 5 \mathrm{AFB} /$ field & $135(47.7)$ & $87(30.7)$ & $22(7.8)$ & $5(1.8)$ & $12(4.2)$ & $9(3.2)$ & \\
\hline
\end{tabular}

${ }^{*} p$ value obtained by chi-square statistic.

TABLE 2: Multinomial analysis of patient's nationality for MTB lineages in southwestern Uganda.

\begin{tabular}{|c|c|c|c|c|c|}
\hline MTB lineage $^{a}$ & Predictors & $B$ coefficients & $p$ value* & Odds ratio ${ }^{\circledR}$ & $95 \% \mathrm{CI}$ \\
\hline \multirow{3}{*}{ Lineage 4 Ugandan family } & Intercept & 2.037 & 0.001 & & \multirow{3}{*}{$0.143-1.758$} \\
\hline & Ugandan & -0.691 & 0.281 & 0.501 & \\
\hline & Non-Ugandan & 0 & - & - & \\
\hline \multirow{3}{*}{ Both lineage 4 and lineage 3} & Intercept & -1.099 & 0.341 & - & \multirow{3}{*}{$0.082-9.203$} \\
\hline & Ugandan & -0.141 & 0.907 & 0.868 & \\
\hline & Non-Ugandan & 0 & - & - & \\
\hline \multirow{3}{*}{ Lineage 3} & Intercept & 1.099 & 0.099 & - & \multirow{3}{*}{$0.075-1.193$} \\
\hline & Ugandan & -1.210 & 0.087 & 0.298 & \\
\hline & Non-Ugandan & 0 & - & - & \\
\hline \multirow{3}{*}{ Unclassified } & Intercept & -1.099 & 0.341 & - & \multirow{3}{*}{$0.130-13.54$} \\
\hline & Ugandan & 0.294 & 0.805 & 1.342 & \\
\hline & Non-Ugandan & 0 & - & - & \\
\hline
\end{tabular}

${ }^{a}$ Reference category is lineage 4 non-Ugandan family; ${ }^{*} p$ value obtained by logistic regression analysis; ${ }^{\circledR}$ adjusted $\mathrm{OR}=$ odds ratio.

found L3 to be at 11\% [28]. Studies conducted in Uganda's neighboring countries such as Tanzania, Sudan, Kenya, and Rwanda have also revealed that L3 (particularly the Central Asian (CAS) family) is a widely implicated lineage in PTB [40]. The MTBC is known to remain stable even in broad cosmopolitan areas such as San Francisco or London where some level of intermingling between locals and immigrants is expected [42]. This is supported by several studies that have shown that MTBC preferably transmits in sympatric host populations $[7,9,11,43]$. Due to these observations, it is thus easy to speculate that different MTBC lineages could have adapted to different human populations, possibly as a result of MTBC's long coevolutionary history and its human host $[6,8,17,18]$ and that local strains are more likely to transmit in a given local setting compared to others. Our observation that $\mathrm{L} 4-\mathrm{U}$ is predominant in this region is consistent with this hypothesis. However, further work is needed to validate this theory, including studies exploring the interaction between MTBC genetic variation and humans. In our study, $6.4 \%$ of the samples were not placed in either of the 
three lineages screened and may warrant their further characterization to better understand them and to add our knowledge to the molecular epidemiology of TB in this area of Uganda.

\section{Limitations of the Study}

The use of three unique SNP markers in this study may have restricted our ability to detect other MTBC lineages causing PTB in this region. Nonetheless, our research used SNP markers validated by a local study and took into account the common lineages in circulation in east and central Africa, where the patient population is from.

\section{Conclusion}

There is heterogeneity of MTBC causing PTB in southwestern Uganda with lineage 4 Ugandan family strains being the most predominant. However, this diversity needs to be ascertained further with more discriminative techniques such as Mycobacterial Interspersed Repeat Units-Variable Number of Tandem Repeat (MIRU-VNTR) typing or whole genome sequencing.

\section{Abbreviations}

$\begin{array}{ll}\text { CAS: } & \text { Central Asian family } \\ \text { DNA: } & \text { Deoxyribonucleic acid } \\ \text { EAI: } & \text { East-African-Indian } \\ \text { L3: } & \text { Mycobacterium tuberculosis lineage } 3 \\ \text { L4: } & \text { Mycobacterium tuberculosis lineage } 4 \\ \text { L4-NU: } & \text { Mycobacterium tuberculosis lineage } 4 \text { other than } \\ & \text { Ugandan family } \\ \text { L4-U: } & \text { Mycobacterium tuberculosis Ugandan family } \\ \text { LAM: } & \text { Latin American-Mediterranean } \\ \text { MTB: } & \text { Mycobacterium tuberculosis } \\ \text { MTBC: } & \text { Mycobacterium tuberculosis complex } \\ \text { PTB: } & \text { Pulmonary tuberculosis } \\ \text { RT-PCR: } & \text { Real-time polymerase chain reaction } \\ \text { SNP: } & \text { Single nucleotide polymorphism } \\ \text { TB: } & \text { Tuberculosis. }\end{array}$

\section{Data Availability}

Information used in the study can be accessed at http://www .re3data.org/.

\section{Ethical Approval}

This was acquired from the Mbarara University of Science and Technology Institutional Review Board committee, and clearance was acquired from the Uganda National Council for Science and Technology Research under reference numbers 13/08-17 and HS2379, respectively.

\section{Conflicts of Interest}

The authors declare that they have no competing interests.

\section{Acknowledgments}

We thank the colleagues from the molecular biology laboratory at Makerere University Medical School and the staff of TB Laboratory of Mbarara University of Science and Technology for the technical assistance.

\section{Supplementary Materials}

Supplementary 1. Figure 1: map of Uganda showing the study area.

Supplementary 2. Table 1: lineage-specific SNP typing primers and probes used in the identification of the MTB lineages using RT-PCR assay.

Supplementary 3. Figure 2: an illustration of the RT-PCR (Bio-Rad CFX96 Touch $^{\mathrm{TM}}$ ) SNP-typing amplicon melting temperature analysis using lineage-specific primers and probes.

\section{References}

[1] R. Firdessa, S. Berg, E. Hailu et al., "Mycobacterial lineages causing pulmonary and extrapulmonary tuberculosis, Ethiopia," Emerging Infectious Diseases, vol. 19, no. 3, pp. 460$463,2013$.

[2] S. Gagneux, "Genetic diversity in Mycobacterium tuberculosis," in Current Topics in Microbiology and Immunology, pp. 1-25, Springer, 2013.

[3] S. Gagneux, "Ecology and evolution of Mycobacterium tuberculosis," Nature Reviews Microbiology, vol. 16, no. 4, pp. 202 213, 2018

[4] E. M. Wampande, P. Naniima, E. Mupere et al., "Genetic variability and consequence of Mycobacterium tuberculosis lineage 3 in Kampala-Uganda," PLoS One, vol. 14, no. 9, article e0221644, 2019.

[5] S. Gagneux and P. M. Small, "Global phylogeography of Mycobacterium tuberculosis and implications for tuberculosis product development," The Lancet Infectious Diseases, vol. 7, no. May, pp. 328-337, 2007.

[6] M. Achtman, "Evolution, population structure, and phylogeography of genetically monomorphic bacterial pathogens," Annual Review of Microbiology, vol. 62, no. 1, pp. 53-70, 2008.

[7] I. Mokrousov, "Mycobacterium tuberculosis phylogeography in the context of human migration and pathogen's pathobiology: Insights from Beijing and Ural families," Tuberculosis, vol. 95, pp. S167-S176, 2015.

[8] S. Gagneux, "Host-pathogen coevolution in human tuberculosis," Philosophical Transactions of the Royal Society B: Biological Sciences, vol. 367, no. 1590, pp. 850-859, 2012.

[9] S. Gagneux, K. DeRiemer, T. van et al., "Variable hostpathogen compatibility in Mycobacterium tuberculosis," Proceedings of the National Academy of Sciences of the United States of America, vol. 103, no. 8, pp. 2869-2873, 2006.

[10] I. Filliol, J. R. Driscoll, D. van Soolingen et al., "Global distribution of Mycobacterium tuberculosis spoligotypes," Emerging Infectious Diseases, vol. 8, no. 11, pp. 1347-1349, 2002.

[11] Z. Rahim, K. Zaman, A. G. M. van der Zanden et al., "Assessment of population structure and major circulating phylogeographical clades of Mycobacterium tuberculosis complex in Bangladesh suggests a high prevalence of a specific subclade 
of ancient M. tuberculosis genotypes," Journal of Clinical Microbiology, vol. 45, no. 11, pp. 3791-3794, 2007.

[12] S. Zamani, M. J. Nasiri, M. Haeili et al., "Determination of circulating Mycobacterium tuberculosis strains and transmission patterns among TB patients in Iran, using 15 loci MIRUVNTR," International Journal of Mycobacteriology, vol. 4, p. 119, 2015.

[13] F. Gehre, M. Antonio, F. Faïhun et al., "The first phylogeographic population structure and analysis of transmission dynamics of M. africanum West African 1- combining molecular data from Benin, Nigeria and Sierra Leone," PLoS One, vol. 8, no. 10, pp. e77000-e77013, 2013.

[14] S. N. Niobe-Eyangoh, C. Kuaban, P. Sorlin et al., "Genetic biodiversity of Mycobacterium tuberculosis complex strains from patients with pulmonary tuberculosis in Cameroon," Infection, Genetics and Evolution, vol. 41, no. 6, pp. 2547-2553, 2003.

[15] D. Stucki, D. Brites, L. Jeljeli et al., "Mycobacterium tuberculosis lineage 4 comprises globally distributed and geographically restricted sublineages," Nature Genetics, vol. 48, no. 12, pp. 1535-1543, 2016.

[16] H. Nebenzahl-Guimaraes, S. A. Yimer, C. Holm-Hansen, J. de Beer, R. Brosch, and D. van Soolingen, "Genomic characterization of Mycobacterium tuberculosis lineage 7 and a proposed name: 'Aethiops vetus'," Microbial Genomics, vol. 2, no. 6, article e000063, 2016.

[17] T. Realpe, N. Correa, J. C. Rozo et al., "Population structure among Mycobacterium tuberculosis isolates from pulmonary tuberculosis patients in Colombia," PLoS One, vol. 9, no. 4, p. e93848, 2014.

[18] A. L. Banuls, A. Sanou, N. T. Van Anh, and S. Godreuil, "Mycobacterium tuberculosis: ecology and evolution of a human bacterium," Journal of Medical Microbiology, vol. 64, no. 11, pp. 1261-1269, 2015.

[19] A. Matteelli, K. Lönnroth, D. Mosca et al., "Cameroon's multidrug-resistant tuberculosis treatment programme jeopardised by cross-border migration," European Respiratory Journal, vol. 47, no. 2, pp. 686-688, 2016.

[20] I. Mokrousov, H. M. Ly, T. Otten et al., "Origin and primary dispersal of the Mycobacterium tuberculosis Beijing genotype: clues from human phylogeography," Genome Research, vol. 15, no. 10, pp. 1357-1364, 2005.

[21] I. Comas, E. Hailu, T. Kiros et al., "Population genomics of Mycobacterium tuberculosis in Ethiopia contradicts the virgin soil hypothesis for human tuberculosis in sub-Saharan Africa," Current Biology, vol. 25, no. 24, pp. 3260-3266, 2015.

[22] E. Alirol, L. Getaz, B. Stoll, F. Chappuis, and L. Loutan, "Urbanisation and infectious diseases in a globalised world," The Lancet Infectious Diseases, vol. 11, no. 2, pp. 131-141, 2011.

[23] S. F. Huang, W. J. Su, H. Y. Dou et al., "Association of Mycobacterium tuberculosis genotypes and clinical and epidemiological features - A multi-center study in Taiwan," Infection, Genetics and Evolution, vol. 12, no. 1, pp. 28-37, 2012.

[24] WHO, Global Tuberculosis Report, World Health Organization, 2013.

[25] Norwegian Refugee Council, Global Report on Internal Displacement, Norwegian Refugee Council, 2020.

[26] A. Edwards, Global forced displacement hits record high, The UN Refugee Agency, 2017.

[27] UNHCR, Uganda Country Refugee Response Plan, 2019.

[28] E. M. Wampande, E. Mupere, S. M. Debanne et al., "Longterm dominance of Mycobacterium tuberculosisUganda family in peri-urban Kampala-Uganda is not associated with cavitary disease," BMC Infectious Diseases, vol. 13, no. 1, 2013.

[29] W. Ssengooba, F. G. Cobelens, L. Nakiyingi et al., "High genotypic discordance of concurrent Mycobacterium tuberculosis isolates from sputum and blood of HIV-infected individuals," PLoS One, vol. 10, no. 7, article e0132581, 2015.

[30] L. Nabyonga, D. P. Kateete, F. A. Katabazi et al., "Determination of circulating Mycobacterium tuberculosis strains and transmission patterns among pulmonary TB patients in Kawempe municipality, Uganda, using MIRU-VNTR," BMC Research Notes, vol. 4, no. 1, pp. 4-9, 2011.

[31] J. Bazira, M. Matte, B. B. Asiimwe, and L. M. Joloba, "Genetic diversity of Mycobacterium tuberculosis in Mbarara, south western Uganda," African Health Sciences, vol. 10, no. 4, pp. 306-311, 2010.

[32] Uganda Tuberculosis profile, 2017, https://www.cdc.gov/ globalhivtb/where-we-work/Uganda.

[33] M. O. Nwang, J. Kawuma, and M. G. Nabukenya, Ministry of Health Manual of the National Tuberculosis and Leprosy, Ministry of Health, Republic of Uganda, 2nd edition, 2010, https:// www.who.int/hiv/pub/guidelines/uganda_tb.

[34] Elizabeth Glaser Foundation, Strengthening the tuberculosis and HIVIAIDS response in the southwest region of Uganda (STAR-SW) project, 2015.

[35] B. Miljković-Selimović, B. Kocić, T. Babić, and L. Ristić, "Bacterial typing methods," Acta Fac Medicae Naissensis, vol. 26, no. 4, pp. 225-233, 2009.

[36] B. A. Forbes, N. Banaiee, K. G. Beavis et al., "Laboratory detection and identification of mycobacteria; approved standard," Clinical Lab Stand Institute (CLSI), vol. 28, no. 17, pp. 14-15, 2008.

[37] E. M. Wampande, S. K. Hatzios, B. Achan et al., "A singlenucleotide-polymorphism real-time PCR assay for genotyping of Mycobacterium tuberculosis complex in peri-urban Kampala," BMC Infectious Diseases, vol. 15, no. 1, 2015.

[38] C. Mulenga, I. C. Shamputa, D. Mwakazanga, N. Kapata, F. Portaels, and L. Rigouts, "Diversity of Mycobacterium tuberculosis genotypes circulating in Ndola, Zambia," BMC Infectious Diseases, vol. 10, no. 1, 2010.

[39] E. S. Solo, Y. Suzuki, T. Kaile et al., "Characterization of Mycobacterium tuberculosis genotypes and their correlation to multidrug resistance in Lusaka, Zambia," International Journal of Infectious Diseases, vol. 102, pp. 489-496, 2021.

[40] E. V. Mbugi, B. Z. Katale, E. M. Streicher et al., "Mapping of Mycobacterium tuberculosis complex genetic diversity profiles in Tanzania and other African countries," PLoS One, vol. 11, no. 5, p. e0154571, 2016.

[41] W. A. Githui, A. M. Jordaan, E. S. Juma et al., "Identification of MDR-TB Beijing/W and other Mycobacterium tuberculosis genotypes in Nairobi, Kenya," The International Journal of Tuberculosis and Lung Disease, vol. 8, no. 3, pp. 352-360, 2004.

[42] E. L. Hamblion, A. le Menach, L. F. Anderson et al., "Recent TB transmission, clustering and predictors of large clusters in London, 2010-2012: results from first 3 years of universal MIRU-VNTR strain typing," Thorax, vol. 71, no. 8, pp. 749756, 2016.

[43] R. Stavrum, M. Mphahlele, K. Øvreås et al., "High diversity of Mycobacterium tuberculosis genotypes in South Africa and preponderance of mixed infections among ST53 isolates," Journal of Clinical Microbiology, vol. 47, no. 6, pp. 18481856, 2009. 